\title{
ULF hydromagnetic oscillations with the discrete spectrum as eigenmodes of MHD-resonator in the near-Earth part of the plasma sheet
}

\author{
V. A. Mazur and A. S. Leonovich \\ Institute of Solar-Terrestrial Physics (ISTP), Russian Academy of Science, Siberian Branch, Irkutsk 33, 664033, Russia
}

Received: 9 December 2005 - Revised: 16 February 2006 - Accepted: 23 February 2006 - Published: 3 July 2006

\begin{abstract}
A new concept is proposed for the emergence of ULF geomagnetic oscillations with a discrete spectrum of frequencies $(0.8,1.3,1.9,2.6 \ldots \mathrm{mHz})$ registered in the magnetosphere's midnight-morning sector. The concept relies on the assumption that these oscillations are MHD-resonator eigenmodes in the near-Earth plasma sheet. This magnetospheric area is where conditions are met for fast magnetosonic waves to be confined. The confinement is a result of the velocity values of fast magnetosonic waves in the nearEarth plasma sheet which differ greatly from those in the magnetotail lobes, leading to turning points forming in the tailward direction for the waves under study. To compute the eigenfrequency spectrum of such a resonator, we used a model magnetosphere with parabolic geometry. The fundamental harmonics of this resonator's eigenfrequencies are shown to be capable of being clustered into groups with average frequencies matching, with good accuracy, the frequencies of the observed oscillations. A possible explanation for the stability of the observed oscillation frequencies is that such a resonator might only form when the magnetosphere is in a certain unperturbed state.
\end{abstract}

Keywords. Magnetospheric physics (Magnetotail; MHD waves and instabilities; Plasma sheet)

\section{Introduction}

Since the foundation work by Dungey (1954), the magnetosphere has been regarded as a natural resonator for various types of MHD oscillations. The framework of this perception allowed a number of later works to put forward a hypothesis favouring the existence of magnetosonic-type global eigenmodes whose localisation area occupies a considerable part of the magnetosphere, rendering them to be

Correspondence to: A. S. Leonovich

(leon@iszf.irk.ru) named "cavity modes" (McClay, 1970) or "global" modes (Kivelson and Southwood, 1986; Southwood and Kivelson, 1986). On the Earth's surface these oscillations are supposed to be observed as Alfvén waves excited by magnetosonic eigen-modes. Each of the magnetosonic modes excites its own continuum of Alfvén waves with frequencies equal to this mode's frequency (i.e. the frequency of the driving force) and hence do not depend on the observation point position. The oscillation spectrum, as a whole, should consist of a discrete set of latitude-independent frequencies.

Such oscillations have indeed been registered in reality - both in HF-radar observations (Ruohoniemi et al., 1991; Samson and Harrold, 1992) and by a ground-based magnetometer network (Mathie et al., 1999; Wanliss et al., 2002). They have explicit peaks in the oscillation spectrum at frequencies 1.3, 1.9, 2.6, 3.4 ... mHz. Even lowerfrequency oscillations are sometimes observed, with 0.6$0.8 \mathrm{mHz}$ (Lessard et al., 2003). The oscillations' frequencies almost do not change, either within a single registration interval or from event to event. They are generally registered in the magnetosphere's midnight-morning sector at latitudes $60^{\circ}$ to $80^{\circ}$. This induced the above papers to conclude that the resonant Alfvén oscillations observed on Earth are excited in tailward-elongated closed field lines.

The above-cited theoretical investigations were concerned with only the most general considerations concerning the possible existence of global (cavity) modes. In the process, they used box-type models which bear little resemblance to reality. To compare theory with experiments, more specific and adequate models of the medium should be employed. To date, enough of this type of work has been completed.

Zhu and Kivelson (1989), Lee and Lysak (1991, 1994), Leonovich and Mazur (2001) have carried out calculations of global mode frequencies, assuming the modes to be localised in the dayside magnetosphere. The calculations were done within the framework of a dipole model with a realistic enough distribution of plasma corresponding to

Published by Copernicus GmbH on behalf of the European Geosciences Union. 


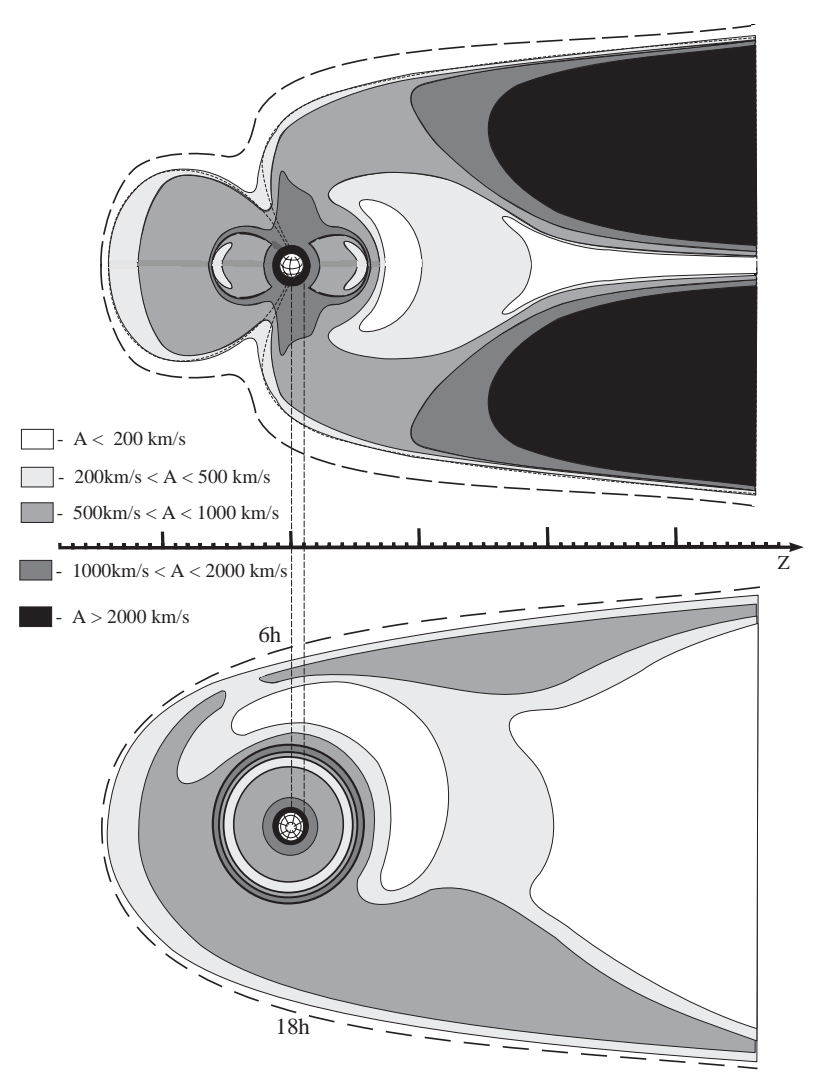

Fig. 1. A sketch of Alfvén velocity distribution in the meridional (a) and equatorial (b) sections of the magnetosphere. Areas with different values of $A$ are shown by varying shades of grey. The NEPS in Fig. 1b has asymmetry due to convective flow of the plasma. An extensive area with a small value of $A$ on the right side of the figure is a section of the plasma sheet in the distant tail. If it deviates from the equatorial plane, this area will be replaced by an area with a greater value of $A$, corresponding to one of the tail lobes.

a moderately disturbed dayside magnetosphere. The frequencies of the eigenmodes are shown to be in the region $f \geq 5 \mathrm{mHz}$. This result is easily understood from simple estimates. In order of magnitude, the frequency of magnetosonic oscillations equals $f \sim A / \lambda$, where $A$ is the typical Alfvén velocity, $\lambda$ is the wavelength. For an eigen-mode $\lambda \gtrsim L$, where $L$ is the typical size of the resonator (if the resonator's sizes differ considerably in the different directions, $L$ is the smallest of them). For the head part of the magnetosphere we have $A \sim 10^{3} \mathrm{~km} / \mathrm{s}, L \sim 20 R_{E} \sim 10^{5} \mathrm{~km}$, whence $f \sim A / L \sim 10 \mathrm{mHz}$, in complete agreement with the results of more precise calculations. One can see that the theoretical values for the frequencies differ too much from the observed ones. But there is an even more important reason not to allow the above-cited works to be regarded as acceptable versions of the global magnetospheric resonator. They ignore the existence of the magnetospheric tail, and therefore the question of whether the eigenmodes are at all confined in the head part of the magnetosphere remains unanswered.
This question has brought to life a series of papers (Harrold and Samson, 1992; Samson and Harrold, 1992; Samson and Rankin, 1994) treating the magnetospheric tail as the region where magnetosonic oscillations with a discrete spectrum are localised. In the meantime, the tail, due to its geometry, is not regarded as a resonator but rather as a waveguide along which generated oscillations can propagate freely. Frequency quantisation in such a waveguide is thought by the above authors to be provided for by the oscillation having the structure of a standing wave along directions transverse to the waveguide, with its wavelength along the waveguide being much larger than across, thereby not affecting the oscillation frequency. The latter suggestion seems too artificial, however. No reasons are apparent as to why the oscillations with a longitudinal wavelength on the order of the transverse wavelength or less cannot be excited. The spectrum of such oscillations is, in the meantime, continuous because the longitudinal wavelength can have any value (is not quantised) and affects the frequency considerably. This difficulty is recognised by the theory's authors (Samson and Rankin, 1994) themselves. Besides, the theoretical oscillation frequency considerably exceeds the observed one in this case as well. Lateral boundaries of such a waveguide are formed by the magnetopause (a 10 to 100-times Alfvén velocity increase in it does produce the effect of reflection of magnetosonic waves). The typical value of the parameter $L \sim(2-3) \cdot 10^{5} \mathrm{~km}$, and the typical value of the Alfvén velocity in the tail lobes (occupying most of its volume) meanwhile is $A \sim(2-3) \cdot 10^{3} \mathrm{~km} / \mathrm{s}$, yielding $f \gtrsim 10 \mathrm{mHz}$.

Another version of the model in question exists where the waveguide is located in the plasma sheet (Siscoe, 1969). But this case also presents the same difficulties with explaining the discrete spectrum and the numerical value of the frequency. In the plasma sheet the typical value of the Alfvén velocity is $A \sim 10^{2} \mathrm{~km} / \mathrm{s}$, while its thickness is $L \sim 2 \cdot 10^{4} \mathrm{~km}$, yielding $f \sim 5 \mathrm{mHz}$. Besides, due to the plasma in the plasma sheet being hot, the typical value of the frequency increases even more, being determined not only by the Alfvén velocity but by the sound velocity in the plasmas as well: $f \sim \sqrt{A^{2}+S^{2}} / L$.

In order to solve convincingly and illustratively the problem of a possible existence of global magnetosonic eigenmodes and their likely locations, we shall consider the global distribution of the Alfvén velocity in the magnetosphere. The most general reasoning implies that the magnetosonic eigenmodes are localised in areas of Alfvén velocity minima. Increased velocity when moving away from the minimum provides conditions for the waves to be reflected, because of an opacity region behind the surface, from which they reflect. In terms of the WKB approximation it is the area of negative values of the squared wave vector. The magnetosonic wave is also reflected from the boundary on which there is a sharp jump in Alfvén velocity. In this case reflection is possible even if $A$ decreases when passing through the boundary, as 
is the case at the magnetopause. The reflection is not complete, with part of the wave's energy penetrating into the solar wind. The portion of the wave's energy passing through the magnetopause is proportional to the ratio of the Alfvén velocities on both sides of the boundary.

Figure 1 shows sketches of the Alfvén velocity isolines in the meridional and equatorial sections of the magnetosphere. They provide a complete enough representation of the threedimensional distribution of $A$. These figures rely on wellknown data concerning the shape, sizes, magnetic field and density of plasma in the major structural components of the magnetosphere - the magnetopause, plasmasphere, external magnetosphere, plasma sheet, tail lobes (see, for example, Sergeev and Tsyganenko, 1980). These figures do not reflect many of the small-scale details in the distribution of $A$, but we assume them to correctly describe its global distribution.

In terms of the problem we are now discussing, the most important constituent in Fig. 1 is the large-scale and deep minimum of $A$ in the near-Earth part of the plasma sheet (NEPS). Figure 1 shows beyond a doubt that it is in this area that the possible existence of a resonator for globalscale magnetosonic waves should be investigated first. Such inspection at a qualitative-analysis level has recently been done by the authors (Leonovich and Mazur, 2005). Note that Fig. 1 exhibits two more areas with rather small values of $A$. The first one is the magnetopause-adjacent outer part of the dayside magnetosphere. It has modes localised in this area which were studied in Zhu and Kivelson (1989); Lee and Lysak (1991, 1994); Leonovich and Mazur (2001). As was mentioned above, $f>10 \mathrm{mHz}$ in this area. It is possible that this resonator adjoins the resonator in the NEPS, thus forming its comparatively small-scale portion. This portion's eigenmodes are part of the upper harmonics of the resonator in the NEPS with quantum numbers $n>5-10$. Meanwhile, it should be realised that the bulk of such a mode with $n>5-10$, specifically the bulk of its energy, lies in the NEPS.

Secondly, a torus-shaped area with minimum Alfvén velocity is located in the equatorial part of the plasmasphere. This area was first pointed out in Gul'elmi (1970) as a "ring trap for low-frequency waves in the Earth's magnetosphere". Estimates show that $f \gtrsim 50 \mathrm{mHz}$ in this area.

In this paper we theoretically investigate the structure and spectrum of some first harmonics of magnetosonic eigenoscillations in the NEPS. We used a model of Alfvén velocity distribution which, on the one hand, conveys the basic features of the distribution in Fig. 1, while, on the other hand, allows the variables in the oscillation equation to be separated. For this purpose, a parabolic system of coordinates is used where the magnetopause is represented as a paraboloid of rotation. It allows a far enough advance in the analytical solution of the problem, coming to numerical calculations at the final, rather simple, stage.

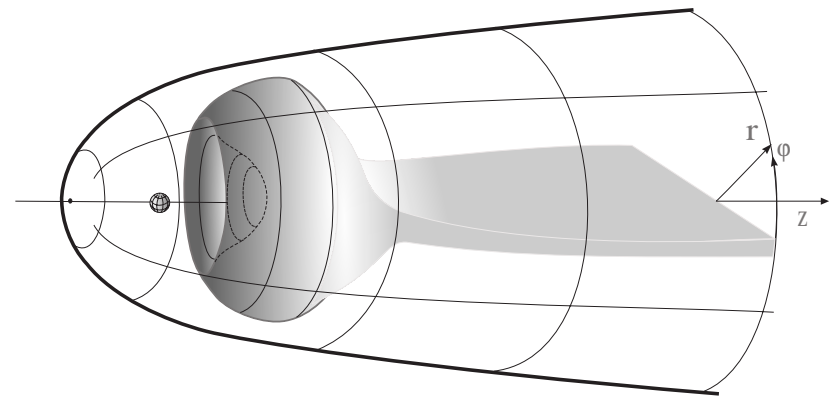

Fig. 2. Model of the medium, and the coordinate system. The plasma sheet is grey. The near-Earth part of the plasma sheet (NEPS) has asymmetry about the $z$ axis. Magnetopause is a paraboloid with its focus in the Earth's centre. Coordinates $(r, z, \varphi)$ form a cylindrical coordinate system.

Section 2 presents a distribution of the Alfvén velocity value in the magnetotail as constructed based on satellite data. Based on these experimental data, an analytical, twodimensional, inhomogeneous model of the magnetospheric plasma distribution is proposed in Sect. 3, which allows one to take into account the main regularities of the Alfvén velocity distribution which are important for the problem under consideration. The basic equations describing the spatial structure and spectrum of magnetosonic oscillations localised in the NEPS are derived. In Sect. 4, the boundary conditions are obtained and the frequency spectrum of the basic harmonics of these oscillations is numerically calculated. In Sect. 5, the calculated spectra are compared to frequencies of ULF oscillations, with discrete spectrum observed in the magnetosphere. Some features of the observed oscillations are discussed in terms of their being interpreted as the resonator eigenmodes in the NEPS. The Conclusion summarises the basic results of this work.

\section{Model of the medium}

The main structural elements of the magnetosphere necessary for our investigation are presented qualitatively in Fig. 2: the magnetospheric boundary (magnetopause) and the plasma sheet, dividing the magnetotail into two lobes. In the near-Earth part of the magnetosphere, the plasma sheet becomes significantly wider, occupying most of the magnetospheric cavity. This is an important feature from the viewpoint of this research.

Note that the velocity distribution of magnetosonic waves in the magnetosphere is of fundamental importance for the problem under study. Velocity of fast magnetosonic waves is represented, in a certain approximation, by $C_{f}=\sqrt{A^{2}+S^{2}}$, where $A=B_{0} / \sqrt{4 \pi \rho}$ is the Alfvén velocity, and $S=\sqrt{\gamma P / \rho}$ is the sound velocity in plasmas. Of much importance for further research is the ratio between the values of $S$ and $A$ in the magnetospheric areas interest. Unlike the Alfvén velocity, 


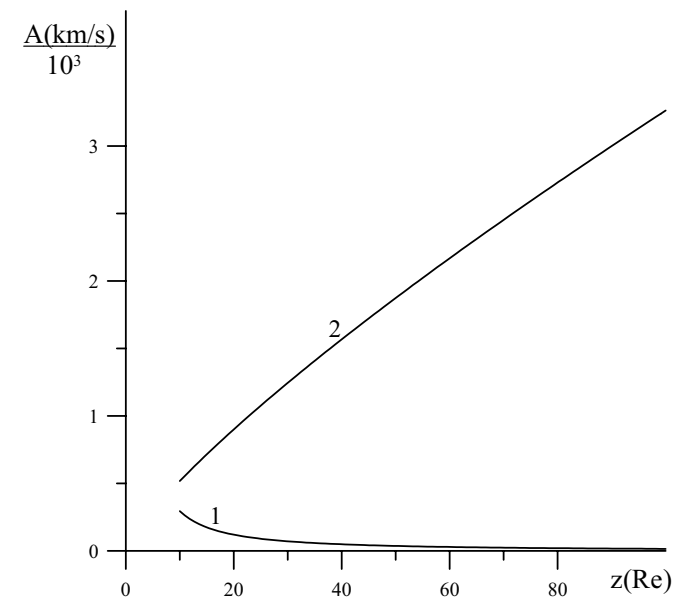

Fig. 3. Average statistical distribution of the Alfvén velocity in the plasma sheet (1) and in the magnetotail lobes (2) in the tailward direction. The distributions are plotted using the approximation formulas in Sergeev and Tsyganenko (1980) for satellite-registered plasma concentration and geomagnetic field intensity. The origin $z=0$ is chosen at the Earth's centre.

the distribution of sound velocity in the magnetosphere is much less known. This is due to sound velocity being a function of temperature, whose distribution in the magnetosphere has been studied much less than the distribution of the magnetic field and plasma density. Since $S^{2} / A^{2} \approx \beta$, where $\beta=8 \pi P / B^{2}$ is the gas-kinetic to magnetic pressure ratio, the distribution of the quantity $\beta$ in the magnetosphere may be examined.

In most of the magnetosphere $\beta \ll 1$, because the magnetosphere is actually a magnetic cavity in the solar wind flow, filled mainly with cold rare plasma. The magnetosphere has two areas where plasma density is comparatively high - the plasmasphere and the plasma sheet. But the plasmaspheric plasma is cold, while the geomagnetic field intensity is high, therefore $\beta \ll 1$ there, as well.

The situation in the plasma sheet is different. Plasma is hot here, whereas the magnetic field intensity is much lower than in the plasmasphere, therefore large values of $\beta$, including $\beta \gg 1$, are possible (Tsyganenko and Mukai, 2003). Due to the importance of this problem, we shall consider it in more detail.

To clearly understand the situation it is necessary to distinguish between the two substantially different parts of the plasma sheet - NEPS and the distant plasma sheet (DPS). Both these parts are filled with nearly the same plasma in terms of density and temperature, so that the pressure in both is approximately the same, as well: $P_{N E P S} \sim P_{D P S}$. Magnetospheric convection causes this plasma to move from DPS to NEPS, where it precipitates into the ionosphere on the inner edge of the plasma sheet. In the process, diffuse aurorae are observed under quiet conditions, with auroral arcs and other active shapes observed under perturbed conditions. But the geomagnetic field configuration in these two parts of the plasma sheet is completely different.

DPS can be regarded as an approximately flat sheet with the magnetic field changing its sign while varying from the value $-B_{T}$ in the tail's one lobe up to $B_{T}$ in the other. Near the central plane of the DPS the magnetic field reduces almost to zero. The equilibrium condition for DPS is $P_{D P S}=B_{T}^{2} / 8 \pi$. It means that plasma pressure in the plasma sheet is balanced by magnetic pressure in the tail lobes. To compute the value of $\beta$ near the central plane of the plasma sheet, one should keep in mind that the magnetic field actually never turns into zero, due to the presence of a comparatively small vertical component $B_{n}$ in the field. The presence of this component results in the plasma sheet magnetic field lines being closed, even though they are very much tailwardelongated. On the order of magnitude $B_{n} \gtrsim 0.1 B_{T}$. Hence, $\beta_{D P S}=8 \pi P_{D P S} / B_{n}^{2}$ is obtained close to the central plane, which is completely with consistent the data in Tsyganenko and Mukai (2003).

The NEPS magnetic field is dipole-like and is strong enough in the entire region, at least $B_{N E P S} \gtrsim B_{T}$. Given that $P_{N E P S} \sim P_{D P S}$, we have $\beta_{N E P S} \gtrsim 1$. The values $\beta_{N E P S} \gg 1$ are impossible. They would mean the existence of plasma in the NEPS whose pressure could not be balanced. The NEPS dimensions, as well as those of the entire magnetosphere, depend more on a magnetic disturbance level. Under quiet conditions, when $K_{P}=1-2$, the NEPS inner edge is approximately $10 R_{E}$, while its external edge is $20-30 R_{E}$ away. When the disturbance is large enough, $K_{P} \gtrsim 5$, these distances decrease to, respectively, $5 R_{E}$ and $10 R_{E}$. The ultra-low-frequency oscillations in which we are interested in are observed only when the disturbance is small, $K_{P}<3$. Therefore, we shall tentatively assume the typical size of the NEPS under these conditions to be $\sim 15 R_{E}=10^{5} \mathrm{~km}$. The value $\beta_{N E P S} \gtrsim 1$ denotes that $S \gtrsim A$ and $C_{f} \sim A$ in this region. Therefore, all qualitative and, on the order of magnitude, quantitative conclusions are possible, if one assumes the plasma to be cold: $C_{f}=A$.

In the plasma sheet, the plasma density is significantly higher, and the Alfvén velocity, respectively, is lower than in the magnetotail lobes. The boundary of the plasma sheet shown in Fig. 2 should be regarded as the boundary dividing the magnetospheric regions, resulting in large and small values of the Alfvén velocity. Therefore this boundary does not reach Earth, near which both geomagnetic field intensity and Alfvén velocity increase.

Figure 3 shows the average statistical tailward distribution of the Alfvén velocity in the magnetotail lobes and in the plasma sheet, plotted from satellite data (Sergeev and Tsyganenko, 1980). The plot draws on approximation formulas proposed in Sergeev and Tsyganenko (1980) for describing the distribution of the concentration $n$ and magnetic field magnitude $B$ in the range of $-10 R_{E}>z>-80 R_{E}$. The formulas $B \approx 3.5(20 / z)^{1.95}(\mathrm{nT})$ and $n \approx(10 / z)^{1.3} \mathrm{~cm}^{-3}$ 
are used for the plasma sheet, and $B \approx 75 / \sqrt{z}(\mathrm{nT})$ and $n \approx(10 / z)^{2.6} \mathrm{~cm}^{-3}$ for the magnetotail lobes, where the $z$ value is expressed in units of the Earth's radius. In this case, $A(\mathrm{~km} / \mathrm{s}) \approx 2.18 B(\mathrm{nT}) / \sqrt{n\left(\mathrm{~cm}^{-3}\right)}$ is the Alfvén velocity. The maximum value of the Alfvén velocity, about $5000 \mathrm{~km} / \mathrm{s}$, is reached in the lobes of the middle part of the magnetotail. Outside the magnetosphere the Alfvén velocity tends to $50-100 \mathrm{~km} / \mathrm{s}$, a value corresponding to the solar wind.

The Alfvén velocity in the magnetotail lobes is more than an order of magnitude higher than in the plasma sheet. As will be shown below, it creates conditions for low-frequency magnetosonic waves to be confined in the NEPS. More detailed satellite measurements of the geomagnetic field intensity and plasma density in the NEPS are presented in Borovsky et al. (1998). Using these data we can determine the following typical values of the Alfvén velocity: $A \approx 240 \mathrm{~km} / \mathrm{s}$ at a tailward distance from Earth $z=-10 R_{E}, A \approx 70 \mathrm{~km} / \mathrm{s}$ when $z=-15 R_{E}$ and $A \approx 200 \mathrm{~km} / \mathrm{s}$ when $z=-25 R_{E}$. Thus, we will assume that the typical value of the Alfvén velocity in the NEPS is close to $200 \mathrm{~km} / \mathrm{s}$.

\section{Coordinate system and main equations}

The spatial structure of the monochromatic fast magnetosonic waves of the type $\sim \exp (-i \omega t)$, where $\omega$ is the wave frequency, is described, in an inhomogeneous magnetosphere, in the cold plasma approximation, by the equation

$\Delta \Phi+\frac{\omega^{2}}{A^{2}} \Phi=0$

where $\Phi$ is any disturbed wave component of the oscillations under study, $A$ is the Alfvén velocity. This equation should be treated as a model. Obtaining an accurate equation for the magnetosonic waves in a 3-D inhomogeneous magnetosphere is rather problematic. Its form depends on both the method of dividing the entire field of MHD oscillations into constituent fields of the Alfvén and magnetosonic waves, as well as on the chosen coordinate system. The coefficients for the derivatives are, of course, different in various curvilinear coordinate systems. When the typical spatial scales of the oscillations under study are much smaller than the typical scale of changes in the medium parameters, however, Eq. (1) describes, with good accuracy, the structure of a magnetosonic wave in any orthogonal coordinate system. At the limit of its applicability, this equation can also be used for oscillations whose wavelength is comparable with the typical scales of the medium inhomogeneity.

The search for a solution to Eq. (1) is significantly simplified if symmetry in some direction is present in the problem. We, in our work, will be interested in the magnetosonic oscillations localised in the NEPS. As follows from Fig. 2, in this part of the magnetosphere there is some axial (about the $z$ axis) symmetry. Plasma sheet geometry in the middle and distant tail breaks this symmetry, of course. But for the problem we discuss, the presence of the distant plasma sheet is inessential, and we can totally neglect it. Both the tail lobes and the distant plasma sheet are opacity regions for the oscillations under examination. Indeed, as follows from Eq. (1), the local dispersion equation $\omega^{2}=\left(k_{x}^{2}+k_{y}^{2}+k_{z}^{2}\right) A^{2}(x, y, z)-$ where $k_{x, y, z}=2 \pi / \lambda_{x, y, z}$ are the local wave numbers, $\lambda_{x, y, z}$ are the local wavelengths on the coordinates $(x, y, z)$ - holds in the WKB approximation for the magnetosonic waves. The value $\bar{A} \approx 200 \mathrm{~km} / \mathrm{s}$ is typical of the NEPS, and for the fundamental harmonics we have $\lambda_{x, y, z} \geq 10^{5} \mathrm{~km}$ (typical wavelengths along all the directions are approximately the same). Thus, for the frequency of fundamental harmonics we have $f=\omega / 2 \pi \approx A / \lambda \approx 0.5 \mathrm{mHz}$. Expressing the squared wave number on the $z$ axis from the local dispersion equation, we obtain

$k_{z}^{2}(x, y, z)=\frac{\omega^{2}}{A^{2}}-\left(k_{x}^{2}+k_{y}^{2}\right)$.

Suppose that there is a transparency region in the NEPS for the low-frequency waves under study. It means that here, $\omega^{2} / A^{2} \gtrsim k_{x}^{2}+k_{y}^{2}$. Moving to the tail lobes the Alfvén velocity increases by an order of magnitude, but the wave numbers $k_{x}^{2}, k_{y}^{2}$, the value of which is determined by the typical scale of the localisation region (in our case, by the typical radius of the geotail cross section), remain practically unchanged. The eventual result is that we have $k_{z}^{2}<0$ in the tail lobes and, thus, they become an opacity region for the low-frequency magnetosonic waves under consideration. It allows us to use an axisymmetric model of the magnetosphere and to translate the initial three-dimensional inhomogeneous problem into a two-dimensional one, which is much simpler for investigation.

Since the magnetospheric cavity resembles a paraboloid, it is natural to choose the parabolic coordinate system to describe it (Madelung, 1961). We will introduce two mutually orthogonal sets of parabolic coordinate surfaces having a common focus at the centre of Earth, denoting them as $\xi$ and $\eta$. These surfaces are obtained by rotating the generatrix parabolas about the $z$ axis, thereby passing through the centres of the Sun and Earth (see Fig. 4). The dimensionless parabolic coordinates $\xi$ and $\eta$ are related to the usual cylindrical coordinates (the $z$ axis being common) by

$$
\begin{aligned}
& \xi=\left(\sqrt{r^{2}+z^{2}}-z\right) / 2 \sigma, \\
& \eta=\left(\sqrt{r^{2}+z^{2}}+z\right) / 2 \sigma,
\end{aligned}
$$

where $\sigma$ is the distance from the focus to the top of the paraboloid $\xi=1$, corresponding to the magnetopause. In other words, $\sigma$ is the distance from the Earth's centre to the subsolar magnetopause point. Since we assume axial symmetry in the problem, we will seek its full solution as an expansion into azimuthal harmonics of the type $\Phi(\xi, \eta, \varphi)=$ $\bar{\Phi}(\xi, \eta) \exp (i m \varphi)$, where $m=0,1,2, \ldots$ is the azimuthal wave 


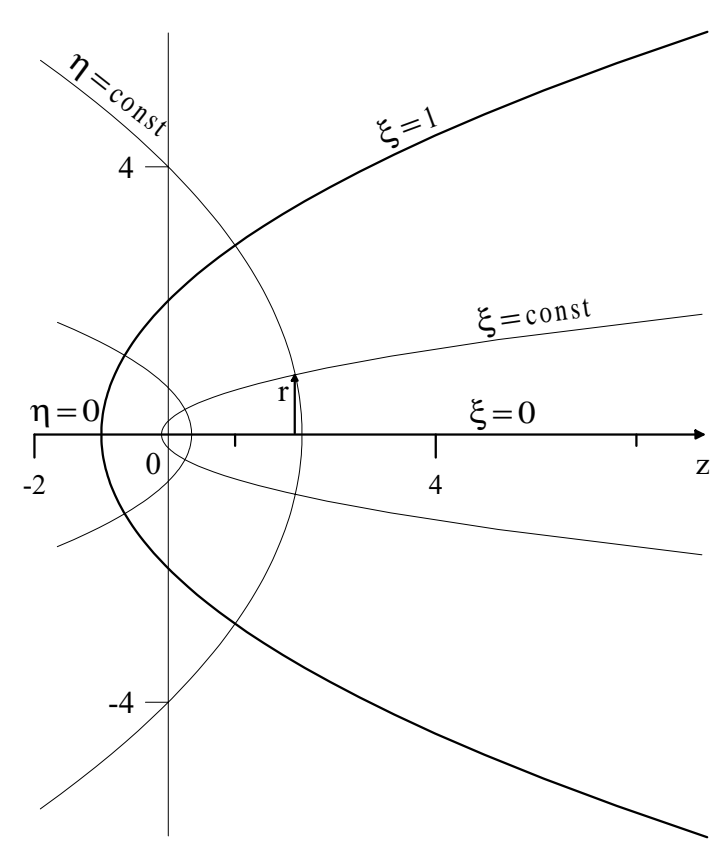

Fig. 4. An orthogonal system of dimensionless parabolic coordinates $(\xi, \eta)$ in a cross section containing the $z$ axis. The focus $z=0$ is located at the Earth's centre. The surface $\xi=1$ coincides with the magnetopause. Semiaxes $z=(0, \infty)$ and $z=(0,-\infty)$ coincide with the coordinate surfaces $\xi=0$ and $\eta=0$, respectively.

number. Equation (1) written in the parabolic coordinates $\xi$ and $\eta$ has the form

$$
\begin{aligned}
\frac{\partial}{\partial \xi} \xi \frac{\partial \bar{\Phi}}{\partial \xi} & +\frac{\partial}{\partial \eta} \eta \frac{\partial \bar{\Phi}}{\partial \eta} \\
+ & {\left[(\xi+\eta) \frac{\omega^{2} \sigma^{2}}{A^{2}(\xi, \eta)}-\frac{m^{2}}{4}\left(\frac{1}{\xi}+\frac{1}{\eta}\right)\right] \bar{\Phi}=0 . }
\end{aligned}
$$

It is easy to verify that if we choose the Alfvén velocity distribution as

$A^{2}(\xi, \eta)=\frac{A_{0}^{2}}{\sigma} \frac{\xi+\eta}{a(\xi)+b(\eta)}$,

where $A_{0}$ is a constant with the dimension of velocity, and $a(\xi)$ and $b(\eta)$ are any functions of the variables $\xi$ and $\eta$, then Eq. (2) becomes an equation with separable variables.

Such functions $a(\xi)$ and $b(\eta)$ have to be chosen as to allow us to simulate the chief regularities of the Alfvén velocity distribution in the magnetospheric region of interest. We will consider the oscillations localised in the magnetosphere, neglecting their small leakage into the solar wind through the magnetopause. Therefore, we will not require correct behaviour from the function $A^{2}(\xi, \eta)$ in the solar wind region when choosing the functions $a(\xi)$ and $b(\eta)$. The major regularities of the Alfvén velocity distribution in the NEPS and in the magnetotail lobes can be modelled by the following functions

$a(\xi)=\left[\alpha_{0}+\left(\alpha_{1}-\alpha_{0}\right) \xi\right] \xi$,

$$
b(\eta)=\left\{\begin{array}{lr}
{\left[\alpha_{0}+\left(\alpha_{2}-\alpha_{0}\right) \eta\right] \eta,} & \eta \leq \eta_{1}, \\
\beta_{1}\left[1-\left(\eta-\eta_{\min }\right)^{2} / \Delta_{1}^{2}\right], & \eta_{1}<\eta<\eta_{2}, \\
\beta_{2}\left[1+\left(\eta-\eta_{\max }\right)^{2} / \Delta_{2}^{2}\right], & \eta \geq \eta_{2},
\end{array}\right.
$$

where $\eta_{\min }$ is the coordinate corresponding to the location of an Alfvén velocity minimum in the NEPS, and $\eta_{\max }$ is the coordinate of a maximum in the middle part of the magnetotail lobes. Parameters of the model (4) are assumed to be chosen such that the function $b(\eta)$ is continuous and smooth. The points $\eta_{1}$ and $\eta_{2}$ can be conventionally considered as the near and far (relative to Earth) boundaries of the NEPS on the $\eta$ coordinate. It is between these points that the turning points of oscillations localised in the NEPS are to be found. The maximum of the Alfvén velocity value in the middle part of the magnetotail serves as a potential barrier for confining low-frequency magnetosonic oscillations in the NEPS. For higher-frequency oscillations this maximum is not a barrier in preventing their tailward escape. This is probably why higher-frequency MHD oscillations with discrete spectrum are not observed in the magnetosphere.

Thus, if the wave function under investigation is presented as $\bar{\Phi}(\xi, \eta)=\psi(\xi) \phi(\eta)$, we can reduce the search for a solution to the initial partial of the differential equation to solving a system of two coupled ordinary differential equations:

$$
\begin{gathered}
\xi \frac{\partial^{2} \psi}{\partial \xi^{2}}+\frac{\partial \psi}{\partial \xi}+\left[\Omega^{2} a(\xi)-\frac{m^{2}}{4 \xi}+Q\right] \psi=0, \\
\eta \frac{\partial^{2} \phi}{\partial \eta^{2}}+\frac{\partial \phi}{\partial \eta}+\left[\Omega^{2} b(\eta)-\frac{m^{2}}{4 \eta}-Q\right] \phi=0 .
\end{gathered}
$$

Here $Q$ is the separation constant, and $\Omega=\omega \sigma / A_{0}$ is the dimensionless frequency. These quantities are the eigenvalues of the problem to be solved.

\section{Results of numerical calculations and their discussion}

Numerically computing the eigenfrequency spectrum of oscillations localised in the NEPS requires constants which determine the parameters of the magnetospheric model to be specified, Eqs. (3), (4). Let us choose the following numerical values for the constants: $A_{0}=750 \mathrm{~km} / \mathrm{s}, \sigma=10 R_{E}$ ( $R_{E}=6370 \mathrm{~km}$ is the Earth's radius), $\alpha_{0}=0.04, \alpha_{1}=10, \alpha_{2}=5$, $\eta_{\min }=1.5, \eta_{\max }=6, \Delta_{1}=1, \Delta_{2}=2.5, \eta_{1}=0.6$, and the $\eta_{2}$ point is found based on the requirement that the second and third expressions in Eq. (4) are equal when $\eta=\eta_{2}$. The appropriate two-dimensional (in a meridional plane) distribution of the Alfvén velocity is shown in Fig. 5. This model describes well enough both the distribution of the Alfvén velocity in the NEPS $\left(\eta_{1}<\eta<\eta_{2}\right)$ and its maximum in the middle tail, as well as demonstrating a qualitatively correct behaviour in the earthward direction.

Additionally, boundary conditions have to be formulated for the functions $\psi(\xi)$ and $\phi(\eta)$, in order to solve the problem. Since, in this calculation, we will not take into account 


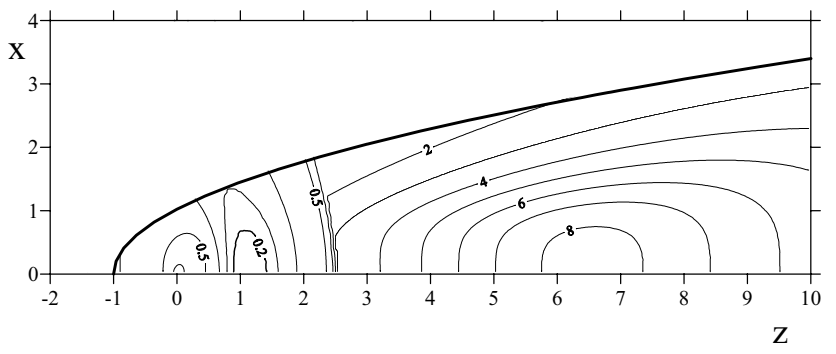

Fig. 5. Isolines of Alfvén velocity value $A\left(10^{3} \mathrm{~km} / \mathrm{s}\right)$ distribution used in the model calculations, in the plane $(x, z)$. Coordinates $x$ and $z$ are in units of $\sigma$ - distance from the Earth's centre to the subsolar point of the magnetopause. The localisation of the NEPS region is $0.6<z<2.5$. The region of the Alfvén velocity maximum in the magnetotail lobes is $z \approx 6.5$.

the small leakage of the oscillations in question into the solar wind, an external boundary condition for the function $\psi(\xi)$ can be formulated at the magnetopause, where $\xi=1$. Let us consider this boundary to be an ideally reflecting wall, which gives us the first boundary condition $\psi(1)=0$. The second boundary condition will be imposed on the symmetry axis: $\xi=0$. As $\xi \rightarrow 0$, the first term in the square brackets in Eq. (5) can be neglected, and the remaining equation reduces to a Bessel equation solved by the Bessel functions:

$\psi(\xi)=C J_{2 m}(2 \sqrt{Q \xi})+D Y_{2 m}(2 \sqrt{Q \xi})$,

where $C$ and $D$ are arbitrary constants. We will require that the solution be finite in its entire domain. Since the function $Y_{v}(x)$ has a singularity as $x \rightarrow 0$, we will assume $D=0$.

A similar boundary condition is obtained for the function $\phi(\eta)$ as $\eta \rightarrow 0$ :

$\phi(\eta)=C I_{2 m}(2 \sqrt{Q \eta})$.

For the oscillations we are discussing, there are turning points, $\bar{\eta}_{1}$ and $\bar{\eta}_{2}\left(0<\bar{\eta}_{1}<\bar{\eta}_{2}<\eta_{2}\right)$, in the WKB approximation, between which they are localised. When $\eta>\bar{\eta}_{2}$, the function $\phi(\eta)$ decreases exponentially in the opacity region. Moving further away tailwards, the Alfvén velocity, having passed its maximum, begins to decrease, and the opacity region is followed again by the transparency region. This creates conditions for the oscillations to partially leak through the potential barrier into the solar wind.

However, if this barrier is wide and high enough, oscillation leakage through it is small (smaller than through the magnetopause), and can be neglected. Thus, the second boundary condition can be formulated in this approximation as an impenetrable wall located deep in the opacity region. If it is located much farther than the typical scale of the function $\phi(\eta)$ 's exponential decay into the opacity region, the solution to be obtained is practically unaffected by where in particular, this wall is located. We, in our calculations, placed it in the region of the Alfvén velocity maximum: $\phi\left(\eta_{\max }\right)=0$.
Table 1. Eigenfrequencies of the MHD resonator in the NEPS $f_{m n l}(\mathrm{mHz})$.

\begin{tabular}{llll}
\hline $\mathrm{m}=0$ & & & \\
\hline $\mathrm{n} \backslash 1$ & 0 & 1 & 2 \\
0 & 0.73 & 1.41 & 2.13 \\
1 & 1.36 & 1.96 & 2.65 \\
2 & 1.97 & 2.55 & 3.2 \\
\hline $\mathrm{m}=1$ & & & \\
\hline $\mathrm{n} \backslash 1$ & 0 & 1 & 2 \\
0 & 1.04 & 1.66 & 2.42 \\
1 & 1.66 & 2.24 & 2.91 \\
2 & 2.29 & 2.84 & 3.47 \\
\hline $\mathrm{m}=2$ & & & \\
\hline $\mathrm{n} \backslash 1$ & 0 & 1 & 2 \\
0 & 1.32 & 1.91 & 2.7 \\
1 & 1.96 & 2.52 & 3.17 \\
2 & 2.59 & 3.13 & 3.75 \\
\hline
\end{tabular}

These boundary conditions are only satisfied by solutions of Eqs. (5), (6) corresponding to certain eigenvalues of the parameters $\Omega=\Omega_{m n l}$ and $Q=Q_{m n l}$, where $m, n, l=0,1,2 \ldots$ - i.e. quantum numbers determining the number of nodes of the desired localised wave function $\Phi_{m n l}(\varphi, \xi, \eta)$ on the coordinates $\varphi, \xi$ and $\eta$, respectively. The set of eigenvalues $\Omega_{m n l}$ determines the set of resonator eigenfrequencies $f_{m n l}=\omega_{m n l} / 2 \pi$. The first three harmonics' eigenfrequencies, for all quantum numbers, found while numerically solving the problem, are presented in Table 1. One can see that the calculated eigenfrequencies tally rather well with the observed frequency spectrum of the magnetospheric low-frequency oscillations with discrete spectrum $(0.8,1.3,1.9,2.6 \ldots \mathrm{mHz})$. There is one interesting feature observed in the spectrum of the calculated frequencies. The resonator eigenfrequencies do not cover the spectrum range evenly, but converge into separate groups. Thus, the frequencies $f_{000}=0.73 \mathrm{mHz}$ and $f_{100}=1.04 \mathrm{mHz}$ represent groups consisting of a single frequency. Each of the frequency groups $\left(f_{001}=1.41 ; f_{010}=1.36 ; f_{200}=1.32 \mathrm{mHz}\right)$ and $\left(f_{101}=1.66 ; \quad f_{110}=1.66 ; \quad f_{300}=1.59 \mathrm{mHz}\right)$ include three harmonics, clustered near the average frequencies $\bar{f} \approx 1.35 \mathrm{mHz}$ and $\bar{f} \approx 1.6 \mathrm{mHz}$, respectively. The other harmonics can be pooled into groups with frequencies $\bar{f} \approx 1.95 \mathrm{mHz}, \bar{f} \approx 2.2 \mathrm{mHz}, \bar{f} \approx 2.6 \mathrm{mHz}, \bar{f} \approx 3.1 \mathrm{mHz}$ ...each group consisting of 5-7 harmonics (including harmonics with quantum numbers $m, n, l>2$ ). Taking into account the finite value of sound velocity $S$ results in corrections to frequencies of the order of $S^{2} / A^{2} \sim \beta$, i.e. for the NEPS conditions - from several percent up to several tens of a percent. 
Other conditions being equal, the ratio between the number of harmonics in the groups can be regarded as a relative probability of observing oscillations with the given average frequency. It is difficult, however, to imagine that the excitation conditions can be the same for oscillations with different spatial structures. This probability is primarily influenced by the presence of a harmonic of given oscillations in the source spectrum. As a source of these oscillations, one could regard, for example, the Kelvin-Helmholtz instability at the magnetopause. The eigenfrequency spectrum of the resonator in question is precisely within the frequency range of oscillations excited by this instability in the magnetotail (Sibeck et al., 1987). These oscillations can also be excited in the magnetosphere under the effect of solar wind pressure impulses (Kepko and Spence, 2003).

\section{Discussion}

Let us discuss some features of ULF oscillations with a discrete frequency spectrum registered in the magnetosphere, regarding them as the eigenmodes of a resonator in the NEPS. Observations imply that these oscillations are chiefly registered in the magnetosphere's midnight-morning sector. The NEPS is known to have noticeable asymmetry relative to the midnight-afternoon meridional plane (Elpic et al., 1999). This is related to the asymmetry of the convective plasma flux from the magnetotail to the dayside, more intense through the morning than through the evening sector of the magnetosphere. The bulk of the NEPS meanwhile shifts into the morning sector and its projection along the field lines onto Earth occupies latitudes between $60^{\circ}-80^{\circ}$. Owing to the eigen-oscillations localised in this part of the plasma sheet, their absence from the dayside and evening magnetosphere receives a natural explanation.

Unfortunately, there is no similar natural and exhaustive explanation for the stability of the registered oscillations' frequencies. Magnetospheric parameters vary within very wide limits, depending on the degree to which the geomagnetic field is disturbed. This is a common difficulty for any existing theoretical interpretations of the observed oscillations. It is possible, however, to try to understand this feature by way of the following reasoning. For an eigen oscillation to set in the resonator, its parameters should remain unchanged for at least several oscillation periods. Since the oscillations in question are sufficiently low-frequency (with periods of about $20 \mathrm{~min}$ ), we can assume that the resonator characteristics remain constant for an hour or more. This is possible only in quiet enough periods in terms of geomagnetic disturbances. As follows from observations, low-frequency oscillations with discrete spectrum are indeed most often registered on a quiet enough geomagnetic background (Mathie et al., 1999). The plasma sheet (at least the NEPS), in the meantime, has probably almost the same parameters, which is what determines the stability of registered frequencies.
When the level of geomagnetic activity rises the plasma sheet characteristics (as well as those of the confining barrier in the tail lobes) lose their stability, and the eigenmodes have sufficient time to form and stand out against a background of changing magnetospheric parameters.

One more observation concerns the oscillations registered on the Earth's surface. It is presumed that the Alfvén waves registered on Earth have been generated on the resonant magnetic shells via the mechanism of field line resonance. The internal part of the magnetosphere adjoining the Earth is an opacity region for the magnetosonic waves under consideration and their amplitude decreases exponentially earthwards (Mathie et al., 1999; Leonovich and Mazur, 2001; Wanliss et al., 2002). Thus, it seems that these oscillations cannot be registered on the Earth's surface proper.

These ideas, however, are only applicable for oscillations with wavelengths that are much smaller than the typical inhomogeneity scales of the magnetospheric plasma. The lowfrequency magnetosonic oscillations we are now discussing have a typical variation scale comparable with the typical scales of magnetospheric inhomogeneity. Therefore, such large-scale magnetosonic oscillations can reach Earth without their amplitude having dropped significantly. On the whole, the issue of the oscillations under study penetrating to the Earth's surface requires further research, including their ground-level polarization.

Damping of the resonator eigenmodes is due to FMSwaves being capable of escaping from the resonator. A part of the resonator boundary is the ring segment of the magnetopause on the magnetosphere flanks. Blanking of the waves on this boundary, as was already mentioned, is forced by their reflection from the Alfvén velocity jump at the magnetopause. But outside the magnetopause, in the solar wind, the Alfvén velocity is less than inside the magnetosphere. In this case the wave propagating inside the magnetosphere can propagate in the solar wind, too. This means that reflection from the magnetopause is partial; the greater the ratio is of the Alfvén velocities in the magnetosphere $A_{m s}$ and in the solar wind $A_{s w}$, the closer the reflectivity to unity. A simple estimation of mode decrement $\gamma$, due to the mode's leakage through the magnetopause, gives $\gamma / \omega \sim A_{s w} / A_{m s}$. The respective values near the magnetopause are $A_{m s} \sim(300-500) \mathrm{km} / \mathrm{s}, A_{s w} \sim(50--100) \mathrm{km} / \mathrm{s}$, hence $\gamma / \omega \sim 0.1-0.3$. The presence of eigenmode damping is known to mean blurring of the eigenfrequency in the oscillation spectrum and its transformation into a spectral peak with a maxima at the eigenfrequency. The typical width of the peak is of the order of $\gamma \sim(0.1-0.3) \omega$. Other possible eigenfrequency spectrum broadening - small-scale spatiotemporal fluctuations of the medium parameters - are unlikely to contribute more than the damping. 


\section{Conclusions}

The main results of this work may be summarised as follows:

1. A new concept is proposed for low-frequency oscillations with discrete spectrum which are registered by HF radars and networks of ground-based magnetometers at high latitudes. The quantisation of oscillation frequencies is supposed to be related to their being eigenmodes of the resonator in the near-Earth plasma sheet (NEPS). A small value of the Alfvén velocity in the NEPS determines the low frequency of the resonator eigen-oscillations. The confinement of the oscillations within the resonator is provided for by the presence of a potential barrier related to the maximum in the distribution of the Alfvén velocity over the middle part of the magnetotail lobes.

2. A resonator frequency spectrum was computed for the NEPS. A model magnetosphere with parabolic geometry was used. A two-dimensional inhomogeneous model of the Alfvén velocity distribution was proposed for the calculations, which takes into account both the minimum in the NEPS and the maximum in the lobes of the mid magnetotail, confining the oscillations in the tailward direction. The eigenfrequencies of such a resonator are shown to form separate groups, whose average frequencies ( $\bar{f} \approx 0.73,1.04,1.35,1.6,1.95,2.2$, $2.6,3.1 \ldots \mathrm{mHz})$ are close to the frequencies of observed oscillations with discrete spectrum $(0.8,1.3,1.9$, $2.6 \ldots \mathrm{mHz})$.

3. Asymmetry in the occurrence of oscillation registration between the midnight-morning and midnight-evening magnetospheric sectors is explained by the asymmetry of the NEPS.

Acknowledgements. This work was partially supported by Russian Foundation for Basic Research grants: RFBR 04-05-64321 and RFBR 06-05-64495, Program of presidium of Russian Academy of Sciences \#16 and OFN RAS \#16.

Topical Editor I. A. Daglis thanks two referees for their help in evaluating this paper.

\section{References}

Borovsky, J. E., Thomsen, M. F., Elphic, R. C., Cayton,T. E., and McComac, D. J.: The transport of plasma sheet material from the distant tail to geosynchronous orbit, J. Geophys. Res., 103, 20 297-20 331, 1998.

Dungey, J. W.: Electrodynamics of the outer atmospheres, Ionos. Sci. Rep., 69, Ionos. Res. Lab., Cambridge, Pa., 1954.

Elphic, R. C., Thomsen, M. F., Borovsky, J. E., and McComas,D. J.: Inner edge of the electron plasma sheet: empirical models of boundary location, J. Geophys. Res., 104, 22 679-22 693, 1999.

Gul'elmi, A. V.: The ring trap for low-frequency waves in the Earth's magnetosphere (in Russian), Pis'ma Zh. Eksp. Teor. Fiz., $12,35-38,1970$.
Harrold, B. G. and Samson, J. C.: Standing ULF modes of the magnetosphere: a theory, Geophys. Res. Let., 19, 1811-1814, 1992.

Kepko, L. and Spence, H. E.: Observation of discrete, global magnetospheric oscillations directly driven by solar wind density variations, J. Geophys. Res., 108, 1257, doi:1029/2002JA009676, 2003.

Kivelson, M. G. and Southwood, D. J.: Resonant ULF waves: a new interpretation, Geophys. Res. Let., 12, 49-52, 1985.

Kivelson, M. G. and Southwood, D. J.: Coupling of global magnetospheric MHD eigenmodes to field line resonances, J. Geophys. Res., 91, 4345-4351, 1986.

Lee, D.-H. and Lysak, R. L.: Monochromatic ULF wave excitation coupling in the dipole magnetosphere, J. Geophys. Res., 96, 5811-5823, 1991.

Lee, D.-H. and Lysak, R. L.: Numerical studies on ULF wave structures in the dipole model, in: Solar Wind Sources of Magnetospheric Ultra-Low-Frequency Waves, Geophys. Monogr. Ser., vol. 81, edited by: Engebretson, M., Takahashi, J. K., and Scholer, M., AGU, Washington, D.C., 293-297, 1994.

Leonovich, A. S. and Mazur, V. A.: Resonance excitation of standing Alfven waves in an axisymmetric magnetosphere (monochromatic oscillations), Planet. Space Sci., 37, 1095$1108,1989$.

Leonovich, A. S. and Mazur, V. A.: On the spectrum of magnetosonic eigenoscillations of an axisymmetric magnetosphere, $\mathrm{J}$. Geophys. Res., 106, 3919-3928, 2001.

Leonovich, A. S. and Mazur, V. A.: Why do ultra-low-frequency MHD oscillations with a discrete spectrum exist in the magnetosphere?, Ann. Geophys., 23, 1075-1079, 2005.

Lessard, M. R., Hanna, J., Donovan, E. F., and Reeves, G. D.: Evidence for a discrete spectrum of persistent magnetospheric fluctuations below $1 \mathrm{mHz}$, J. Geophys. Res., 108(A3), 1125, doi:10.1029/2002JA009311, 2003.

Madelung, E.: Mathematical tools for the physicist, (in Russian), Nauka, Moscow, 275-276, 1961.

Mann, I. R., Wright, A. W., Mills, K. J., and Nakariakov, V. M.: Excitation of magnetospheric waveguide modes by magnetosheath flows, J. Geophys. Res., 104(A1), 333-354, 1999.

Mathie, R. A., Menk, F. W., Mann, I. R., and Orr, D.: Discrete field line resonances and the Alfven continuum in the outer magnetosphere, Geophys. Res. Lett., 26, 659-662, 1999.

McClay, J. F.: On resonant modes of a cavity and the dynamical properties of micropulsations, Planet. Space Sci., 18, 1673-1682, 1970.

Ruohoniemi, J. M., Greenwald, R. A., and Baker, K. B.: HF radar observations of Pc5 field line resonances in the midnight/early morning MLT sector, J. Geophys. Res., 96, 15 697-15 710, 1991.

Samson, J. C. and Harrold, B. G.: Field line resonances associated with waveguides in the magnetosphere, Geophys. Res. Lett., 19, 441-444, 1992.

Samson, J. C., and Rankin, R.: The coupling of solar wind energy to MHD cavity modes, waveguide modes and field line resonances in the Earth's magnetosphere, in: Solar Wind Sources of Magnetospheric Ultra-Low-Frequency Waves, Geophys. Monogr. Ser. vol. 81, edited by: Engebretson, M. J., Takahashi, J. K., and Scholer, M., 253-264, AGU, Washington, D.C., 1994.

Sergeev, V. A. and Tsyganenko, N. A.: The Earth's magnetosphere (in Russian), Moscow, Nauka, 1980.

Sibeck, D. G., Slavin, J. A., and Smith, E. J.: ISEE 3 magnetopause 
crossings - Evidence for the Kelvin-Helmholtz instability, in: Magnetotail physics (A88-46526 19-46), Baltimore, MD, Johns Hopkins University Press, 73-76, 1987.

Siscoe, G. L.: Resonant compressional waves in the geomagnetic tail, J. Geophys. Res., 74, 6482-6486, 1969.

Southwood, D. J. and Kivelson, M. G.: The effect of parallel inhomogeneity of magnetospheric hydromagnetic wave coupling, J. Geophys. Res., 91, 6871-6877, 1986.
Tsyganenko, N. A. and Mukai, T.: Tail plasma sheet models derived from Geotail particle data, J. Geophys. Res., 108, 1136, doi:10.1029/2002JA009707, 2003.

Wanliss, J. A., Rankin, R., Samson, J. C., and Tikhonchuk, V. T.: Field line resonances in a stretched magnetotail: CANOPUS optical and magnetometer observations, J. Geophys. Res., 107, SMP9,1100, doi:10.1029/2001JA000257, 2002.

Zhu, X. and Kivelson, M. G.: Global mode ULF pulsations in a magnetosphere with a nonmonotonic Alfven velocity profile, J. Geophys. Res., 94, 1479- 1485, 1989. 